\title{
Correction to: Influence of environmental factors on bird diversity in greenspaces in an Amazonian city
}

\author{
José F. Rico-Silva ${ }^{1}$ Emmy J. Cruz-Trujillo ${ }^{1}$ • Gabriel J. Colorado Z. ${ }^{2}$
}

Published online: 18 August 2020

(C) Springer Science+Business Media, LLC, part of Springer Nature 2020

\section{Correction to: Urban Ecosystems}

https://doi.org/10.1007/s11252-020-01042-x

The original version of this article unfortunately contained errors.

Table 2 is incomplete. The corrected Table 2 is presented in this article.

The online version of the original article can be found at https://doi.org/ 10.1007/s11252-020-01042-x

José F. Rico-Silva

josefabiorico@gmail.com

1 Grupo de Investigación en Fauna Silvestre, Universidad de la Amazonia, Florencia 180002, Caquetá, Colombia

2 Departamento de Ciencias Forestales, Universidad Nacional de Colombia, Medellín 050034, Antioquia, Colombia 
Table 2 Bird species detected in the 18 greenspaces of the city of Florencia, including abundance and the number of sites where the species was recorded, May-June 2017. Bird taxonomy follows Remsen et al. (2019)

\begin{tabular}{|c|c|c|c|}
\hline Family & Species & Abundance & No. of sites \\
\hline \multirow[t]{4}{*}{ Columbidae } & Columba livia & 202 & 12 \\
\hline & Columbina talpacoti & 69 & 18 \\
\hline & Leptotila rufaxilla & 4 & 3 \\
\hline & Patagioenas cayennensis & 35 & 15 \\
\hline \multirow[t]{2}{*}{ Cuculidae } & Crotophaga ani & 12 & 2 \\
\hline & Coccyzus melacoryphus & 1 & 1 \\
\hline \multirow[t]{3}{*}{ Trochilidae } & Chionomesa fimbriata & 12 & 9 \\
\hline & Anthracothorax nigricollis & 9 & 6 \\
\hline & Chlorostilbon mellisugus & 1 & 1 \\
\hline Charadriidae & Vanellus chilensis & 1 & 1 \\
\hline Ardeidae & Butorides striata & 2 & 1 \\
\hline Threskiornithidae & Phimosus infuscatus & 1 & 1 \\
\hline Cathartidae & Coragyps atratus & 49 & 15 \\
\hline Accipitridae & Rupornis magnirostris & 5 & 5 \\
\hline \multirow[t]{2}{*}{ Strigidae } & Asio stygius & 2 & 2 \\
\hline & Glaucidium brasilianum & 1 & 1 \\
\hline \multirow[t]{2}{*}{ Picidae } & Melanerpes cruentatus & 3 & 2 \\
\hline & Picumnus lafresnayi & 1 & 1 \\
\hline \multirow[t]{6}{*}{ Psittacidae } & Amazona amazonica & 1 & 1 \\
\hline & Amazona ochrocephala & 6 & 2 \\
\hline & Ara severus & 2 & 1 \\
\hline & Aratinga weddellii & 1 & 1 \\
\hline & Orthopsittaca manilatus & 12 & 2 \\
\hline & Psittacara leucophthalmus & 3 & 2 \\
\hline Furnariidae & Dendroplex picus & 3 & 2 \\
\hline \multirow[t]{13}{*}{ Tyrannidae } & Empidonax alnorum & 4 & 3 \\
\hline & Legatus leucophaius & 1 & 1 \\
\hline & Machetornis rixosa & 8 & 3 \\
\hline & Megarynchus pitangua & 1 & 1 \\
\hline & Myiarchus ferox & 4 & 3 \\
\hline & Myiodynastes maculatus & 1 & 1 \\
\hline & Myiozetetes similis & 19 & 11 \\
\hline & Phaeomyias murina & 21 & 11 \\
\hline & Pitangus sulphuratus & 47 & 17 \\
\hline & Pyrocephalus rubinus & 5 & 4 \\
\hline & Todirostrum cinereum & 16 & 10 \\
\hline & Tyrannus melancholicus & 32 & 16 \\
\hline & Tyrannus tyrannus & 1 & 1 \\
\hline Vireonidae & Vireo olivaceus & 1 & 1 \\
\hline Corvidae & Cyanocorax violaceus & 2 & 2 \\
\hline \multirow[t]{2}{*}{ Hirundinidae } & Progne tapera & 1 & 1 \\
\hline & Pygochelidon cyanoleuca & 5 & 2 \\
\hline \multirow[t]{2}{*}{ Troglodytidae } & Campylorhynchus turdinus & 7 & 3 \\
\hline & Troglodytes aedon & 29 & 14 \\
\hline Turdidae & Turdus ignobilis & 25 & 15 \\
\hline \multirow[t]{7}{*}{ Thraupidae } & Paroaria gularis & 2 & 1 \\
\hline & Ramphocelus carbo & 51 & 17 \\
\hline & Sicalis flaveola & 88 & 18 \\
\hline & Sporophila nigricollis & 3 & 1 \\
\hline & Tangara mexicana & 18 & 6 \\
\hline & Thraupis episcopus & 58 & 18 \\
\hline & Thraupis palmarum & 38 & 15 \\
\hline Passerellidae & Ammodramus aurifrons & 2 & 1 \\
\hline Cardinalidae & Piranga olivacea & 1 & 1 \\
\hline \multirow[t]{5}{*}{ Icteridae } & Cacicus cela & 10 & 6 \\
\hline & Molothrus bonariensis & 11 & 4 \\
\hline & Molothrus oryzivorus & 3 & 2 \\
\hline & Psarocolius angustifrons & 7 & 3 \\
\hline & Psarocolius decumanus & 11 & 4 \\
\hline \multirow[t]{2}{*}{ Fringillidae } & Euphonia laniirostris & 24 & 9 \\
\hline & Euphonia xanthogaster & 4 & 2 \\
\hline
\end{tabular}

